\title{
0391 SLIPPING AS OUTCOME: IMPLICATIONS FOR EPIDEMIOLOGIC RESEARCH ON FALL-RELATED INJURIES
}

TK Courtney*, S K Verma, YHHuang, WR Chang, KW Li, A J Filiaggi Correspondence: Center for Injury Epidemiology, Liberty Mutual Research Institute for Safety, 71 Frankland Road, Hopkinton, MA, 01748, USA

\subsection{6/ip.2010.029215.391}

One major challenge to the study of occupational injuries in active work environments is the rarity of the outcome. Slipping, tripping and falling are responsible for a substantial injury burden in the global workplace. Slipping is an important precursor to falling and subsequent injury though not every slip results in a fall or injury. We applied epidemiologic methods to study individual and work environment factors related to self-reported slipping in US fast food restaurant workers.

Ten limited service restaurants in the Northeastern USA were recruited to participate. Workers occupational slip and/ or fall history within the past 4 weeks was collected along with age, gender, job tenure, work hours per week and work. Shoe type, condition and gross shoe contamination were visually assessed. Floor friction was measured. The logistic generalised estimating equations model was used to compute adjusted ORs.

126 workers (60\% female, mean age 30) participated. Multivariable regression showed that higher restaurant mean coefficient of friction (COF) was significantly associated with a decreased risk of self-reported slipping in the past 4 weeks (OR $0.59,95 \%$ CI 0.42 to 0.82 ). From the highest to the lowest COF restaurant, the odds of a positive slip history increased more than seven times. Younger age, male gender, lower weekly work hours and the presence of gross contamination on workers shoe sole were also positively associated with slipping.

Using self-reported slipping as an upstream measure of outcome may make assessment of risk factors and interventions for fall-related injuries more feasible. 